\title{
Ensemble prediction study of the East Australian Current
}

\author{
T. J. O'Kane ${ }^{1} \quad$ P. R. Oke $^{2} \quad$ P. A. Sandery ${ }^{3}$
}

(Received 13 December 2010; revised 14 April 2011)

\begin{abstract}
We examine the role of dynamical instabilities and flow dependent, growing errors in an ensemble prediction study of the East Australian Current. In the region of the Tasman front where the East Australian Current separates from the coast, the circulation is characterised by meandering and significant mesoscale eddy variability, and is dominated by nonlinear dynamics, thereby representing a severe challenge for operational forecasting. We explore the mechanisms for and structures of forecast errors over a month using the Australian operational ocean forecast system. To examine the role of dynamical instabilities, forecast ensemble perturbations are generated as bred vectors allowing the identification of those perturbations to a given initial state that grow most rapidly. We consider a period spanning the Austral autumn/summer corresponding to the seasons of maximum eddy variability. We find that the bred vectors span a low dimensional subspace whose structures correspond to regions of large forecast error. Further, these structures typically occur in regions of instability and in particular in the vicinity
\end{abstract}

http://anziamj . austms.org.au/ojs/index.php/ANZIAMJ/article/view/3779 gives this article, (c) Austral. Mathematical Soc. 2011. Published April 21, 2011. ISSN 1446-8735. (Print two pages per sheet of paper.) Copies of this article must not be made otherwise available on the internet; instead link directly to this URL for this article. 
of the Tasman front and the East Australian Current extension. Our results suggest that even a very few bred vectors will be useful in adaptive sampling and targeted observations.

\section{Contents}

1 Introduction

2 East Australian Current dynamics

3 Model description

4 Ensemble prediction

5 Results

6 Discussion and conclusions

References

\section{Introduction}

Ocean forecasting has seen major advances in the past decade with many countries now routinely performing operational forecasts of the mesoscale ocean circulation [4]. Many of the advances in ocean forecasting are on the back of numerical weather prediction, particularly those in data assimilation. Ocean forecasting is underpinned by satellite observations of sea level anomalies and sea surface temperature (SST), and in situ observations from Argo floats and a sparse array of tropical moorings. The most energetic scales of the oceans are in the mesoscale, which is characterised by eddies and meanders, and occurs particularly in western boundary current regions. These scales are 
only marginally resolved by the above mentioned components of the global ocean observing system. As a result, we expect the errors of operational ocean forecasts to be variable in time. The errors of the day are likely to depend on the coverage of assimilated observations and the stability of the ocean's circulation while the respective roles of model and initialisation errors in ocean forecasting remains a largely open question.

A primary aim in ensemble prediction is to generate independent initial perturbations as fast growing disturbances with structures and growth rates typical of the analysis errors, that is the perturbation vectors span the possible errors in the control analysis. In contrast, random initial perturbations sampled isotropically, grow more slowly and in some cases even decay leading to underestimated error variances. Our approach to ensemble prediction for the mesoscales of the East Australian Current (EAC) is based on generating an ensemble of deterministic forecasts whose differences are perturbation vectors, centred about an unperturbed or control forecast. The initial perturbation vectors are typically chosen to capture flow dependent information about the deterministic chaotic system [11]. The computational cost of running many realisations of an eddy resolving ocean model leads to severe restrictions on the number of available perturbations and so we aim to sample only the fastest growing error structures using a very small ensemble (four members) of bred vectors (BVs) [14] to qualitatively represent the errors of the day. Bred, Lyapunov and singular vectors (to the tangent linear operator) [11] may all be applied to identify the spatial structures of a system's fastest growing modes; however, because of their inherent linearity, singular and Lyapunov vectors may be inappropriate for systems whose errors grow nonlinearly.

O'Kane et al. [8] showed that a small ensemble of bred vectors (up to twelve members) can provide useful prognostic information about where regions of large forecast or background errors may occur in the EAC over a period characteristic of a typical ocean data assimilation window (seven days). In the present study we seek to determine whether this methodology can improve the predictive skill of an operational ocean forecast system in monthly forecasts. 
Section 2 discusses EAC dynamics and Section 3 our regional ocean model. The ensemble prediction methodology and experimental design are described by Section 4, while the results, a discussion and conclusions are presented in Sections 5 and 6.

\section{East Australian Current dynamics}

The main features of the Tasman Sea are the EAC and its eddies. The EAC forms near $15^{\circ} \mathrm{S}$, and flows along the coast carrying on average $22 \mathrm{~Sv}$ $\left(1 \mathrm{~Sv}=10^{6} \mathrm{~m}^{3} \mathrm{~s}^{-1}\right)$, attaining its maximum volume transport at $30^{\circ} \mathrm{S}$. The EAC tends to separate from the coast near Sugarloaf Point (SPT in Figure 1) $\left(32.5^{\circ} \mathrm{S}\right)$, where it forms a complicated field of warm- and cold-core eddies in a region known as the Tasman front, before flowing southeastward into the Tasman Sea [5].

Warm-core eddies are typically large, with diameters of several hundred kilometers. Cold-core eddies are smaller, perhaps $50-100 \mathrm{~km}$ across, and often form at the point where the EAC separates from the coast, or on the peripheries of warm-core eddies [10]. While warm-core eddies are usually well resolved by altimetry, cold-core eddies are often missed. We therefore expect that forecasting the development of cold-core eddies is problematic, and is unlikely to be skilful. To further complicate the picture, the EAC periodically exhibits a strong extension along the coast to the Southernmost point of Tasmania (EAC extension) with an associated reduction of transport along the Tasman front.

Recent studies using remotely sensed data and ocean models [6] characterised EAC eddy variability as originating via intrinsic instabilities of the current, and showed that eddy shedding events occur on a timescale of about 90 days between $32^{\circ} \mathrm{S}$ and $35^{\circ} \mathrm{S}$. Further, these events were typically preceded by a southward extension of the current beyond $32^{\circ} \mathrm{S}$ to as far as $34^{\circ} \mathrm{S}$, and showed that post shedding the current becomes more strongly zonal at the 

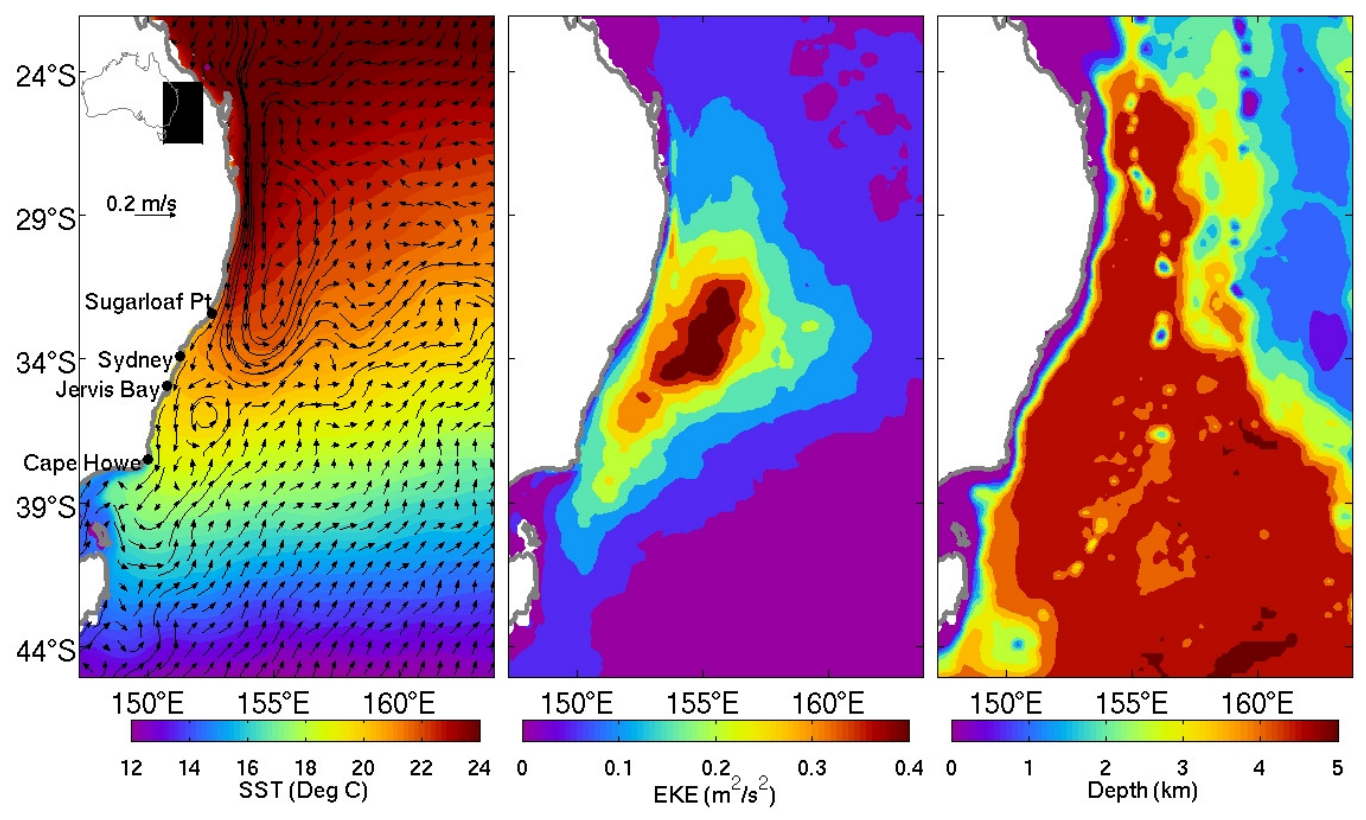

FiguRE 1: Thirteen year average (1993-2006) SST and surface velocities (left), eddy-kinetic energy (EKE; middle) computed from daily mean fields of surface velocity [13], and model bathymetry (right). The inset on the left panel shows the location of the region of interest off south eastern Australia. 
separation point (SPT). More generally, the respective roles of barotropic and baroclinic instability in the initiation and development of EAC eddies is not well understood.

\section{Model description}

The ensemble prediction studies presented Section 5 utilise analyses provided by the Australian Bureau of Meteorology's Ocean Model Analysis and Prediction System (OCEANMAPS [1]) using an eleven day window of observations that include sea surface height anomaly (SSH) from altimetry, temperature and salinity profile observations from XBT, CTD and Argo, and AMSRE SST. The model we use is a regional version of MOM4p1 [3]. It has $0.1^{\circ}$ horizontal resolution and $47 z$-levels with $10 \mathrm{~m}$ vertical resolution in the upper $200 \mathrm{~m}$, expanding to larger spacing towards full oceanic depths. The regional model is nested inside the global model and uses sponge layer open boundary conditions for surface height $\eta$, temperature $T$, salinity $S$, and the $u$ and $v$ components of the velocity field, and uses the same grid and bathymetry as the global model. The domain chosen for this study covers an area in the Tasman Sea enclosed within $148.75^{\circ} \mathrm{E}$ to $163.75^{\circ} \mathrm{E}$ and $45.05^{\circ} \mathrm{S}$ to $22.05^{\circ} \mathrm{S}$ (see Figure 1). The regional model is forced by surface fluxes from the analysis numerical weather prediction cycle that is run operationally by the Australian Bureau of Meteorology. We employ the dynamical initialisation scheme of Sandery et al. [12] that applies time and space dependent tendency forcing as a function of differences between model and target prognostic variables, thereby maintaining dynamical balance.

\section{Ensemble prediction}

The reasons that deterministic forecasts fail over reasonable prediction periods was found to be in large part due to the inherent non-linearity in the system, 
errors in the initial conditions (analysis errors [9]), model deficiencies such as those arising due to poor or inaccurate subgrid scale parameterizations [7], and forcing errors. In comparison to a single control forecast, ensemble forecasts can provide not only improved estimates of the forecast (ensemble mean), but also estimates of the forecast error covariance and possibly the higher order moments.

We postulate that for regions of large eddy variability, such as the EAC, forecast errors at lead times from a week to a month should largely arise due to local dynamic instability processes. Our approach is to generate periodically an initial analysis using OCEANMAPS, about which an ensemble is constructed by adding to the initial analysis finite amplitude growing perturbation vectors that are dynamically generated using the breeding technique. The ensemble, including an unperturbed control or background forecast, is then integrated forward and the evolved perturbation vectors compared to the forecast error which is taken to be the difference between the control forecast and a verifying analysis. The choice of initial perturbation amplitude and period between rescaling sets the spatio-temporal scales of interest, thus the BV approach potentially allows one to focus on dynamical instabilities with a range of growth rates and saturation amplitudes. Our EAC bred vectors are norm dependent in that there is little evidence of convergence onto the leading Lyapunov vector, therefore we must be careful in choosing the size of our initial perturbation and rescaling norm.

We wish to excite processes associated with baroclinic instabilities with error growth rates and structures appropriate to the control analysis errors over a given time window. On timescales of a month or less the dominant EAC instabilities arise due to baroclinic instability associated with mesoscale eddy formation. These instabilities are shown to be centred on or about the thermocline, which is at approximately $250 \mathrm{~m}$ depth. Through experimentation we found that introducing a small uniform, random, spatially uncorrelated perturbation to the temperature $\mathrm{T}$ to be the most appropriate means of ensemble initialisation prior to breeding. Subsequent rescaling against $\mathrm{T}$ at $250 \mathrm{~m}$ (т250) (or equivalently salinity at $250 \mathrm{~m}$ or $\eta$ ) was found to be an 
effective method of perturbing the thermocline.

The procedure for computing $n$ bred vectors follows.

1. First we add $n$ random fields to the analysis temperature fields at all levels. In practice we apply a small uniformly distributed random perturbation to $T$ at all horizontal grid points and at all vertical levels so that the perturbed field is $T_{\mathfrak{n}}^{p}\left(t_{0}\right)=T^{a}\left(t_{0}\right)+\delta T_{n}$ where $p$ denotes perturbation, $a$ analysis, $t_{0}$ is the initial time and where $\delta T$ is a three dimensional field of spatially uncorrelated random numbers.

2. Next the model is integrated forward for $\delta t$ days for $n$ perturbed fields and for an additional unperturbed control forecast $\mathrm{T}^{\mathrm{c}}\left(\mathrm{t}_{0}\right)=\mathrm{T}^{\mathrm{a}}\left(\mathrm{t}_{0}\right)$.

3. After integration, the difference between the $n$ perturbed forecasts $T_{\mathfrak{n}}^{p}\left(t_{0}+\delta t\right)$ and the control forecast $T^{f}\left(t_{0}+\delta t\right)$ is calculated and scaled using the $L_{2}$ or Euclidean norm. Specifically the rescaling factor $f_{n}$ is determined as the ratio of initial anomaly RMS over evolved anomaly RMS at T250 for each $n$ perturbed forecasts. This rescaling factor is now applied globally to all perturbation fields and at all model levels, for all prognostic variables. We now have $n$ perturbation vectors $P_{n}$ for each field $\mathrm{F}_{i=1, \ldots, 5} \in(\eta, T, S, u, v)$,

$$
P_{n}\left(F_{i_{n}}, t_{0}+\delta t\right)=f_{n}\left(t_{0}+\delta t\right)\left[F_{i_{n}}^{p}\left(t_{0}+\delta t\right)-F_{i}^{f}\left(t_{0}+\delta t\right)\right],
$$

where the superscripts $p$ and $f$ denote perturbation and forecast respectively.

4. The $n$ rescaled perturbation vectors are now added to the current analysis provided by the OCEANMAPS data assimilation system and the new ensemble is integrated forward using the full primitive equation ocean model.

5. Repeat steps 2, 3 and 4 noting that we now have $\mathrm{n}$ perturbed fields for (SSH $\eta$, temperature $T$, salinity $S$, and velocity fields $u$ and $v$ ).

After an initial transient period ( $\mathrm{N} \approx 8$ weeks or two cycles) clearly defined growing perturbation vector structures develop. In our experiments we 
consider a maximum of $n=4$ ensemble members. We take the growth over an analysis period (28 days) to be the inverse of the bred vector rescaling parameter at the analysis time in Corazza et al. [2]. We chose to use ensemble averaging of unpaired BVs as a means for determining the local low dimensional subspace spanned by the BVs; we refer to this subspace as regions where BVs align. In the subsequent results and discussion sections we take the difference between the day 28 control (unperturbed) forecast and the verifying analysis to be the control forecast error.

\section{$5 \quad$ Results}

In trying to predict the dynamics of the EAC we are interested in where the BVs align as determined by the subspace where local instabilities are dominant. Figure 2 (rows 1 and 2) shows the verifying analysis and the day 28 forecast valid for the same date. In (rows 3 and 4) an ensemble average of four evolved (day 28) BVs is compared to the control forecast error (row 3; shaded) for sea level after three successive 28 day periods beginning on the 12th February 2008 and valid on the 11th March, and 8th April respectively. Having determined areas where the bred vectors align and have significant amplitude, we are interested to see if these areas correspond to regions of large forecast error. Figure 2 (row 4) shows individual BVs (red, blue, green, magenta) at the $0.4 \mathrm{~m}, 0.25 \mathrm{~m}$ and $0.15 \mathrm{~m} \eta$ contours. The corresponding ensemble average was superimposed on the forecast errors (Figure 2, row 3, black contours). Through their diversity the individual BVs illustrate the inherent nonlinearity of the EAC with little evidence of collapse onto the leading Lyapunov vector. The BVs align in regions corresponding to the dominant dynamic instabilities and in particular are largest in the region of the Tasman front centred offshore of $\mathrm{SPT}\left(32.5^{\circ} \mathrm{S}\right)$, and that these regions are the areas of the largest forecast errors.

The evolving BVs and their corresponding growth factors (not shown) describe the growth and decay of dynamic instabilities and areas of large forecast un- 
certainty. Clearly day 28 forecast errors are dominated by the eddy variability of the EAC and coherent areas of large forecast error associated with fast growing instabilities appear to be captured by the BVs. The EAC exhibits constantly evolving instabilities that undergo rapid growth and decay cycles. These instabilities are associated with eddy variability in a band extending west from $32^{\circ}$ to $35^{\circ} \mathrm{S}$. This behaviour is seen in the evolution of the $\mathrm{BV}$ averages in Figure 3 which plots T250 over a three month period beginning 12th February 2008. These figures show the evolved BVs at day 28, showing the $\pm 3.5, \pm 2.0$ and \pm 1.0 contours, and the forecast error (colour). Figure 3 further demonstrates significant alignment and anti-correlation of the BVs to the forecast errors over the entire three month period shown, and in particular captures the transition between a dominant Tasman front (Figure 3 a) with the current separating from the coast at SPT $\left(32.5^{\circ} \mathrm{S}\right)$, and one where the EAC extension dominates (Figure $3 \mathrm{c}$ ) with the current following the coast down to $45^{\circ} \mathrm{S}$.

We next examine temperature and salinity through the water column in vertical sections (Figures 4 and 5, respectively). We focus on a the particular case of the verification of a 28 day forecast valid for the 8th April when the EAC extension is the dominant feature of the circulation. Comparing Figures $4(\mathrm{a}-\mathrm{c})$ and $5(\mathrm{a}-\mathrm{c})$ we see the remarkable similarity of the spatial structures between SSH and T250. The $\mathrm{T}$ and $\mathrm{S}$ forecast errors and bred vectors are strongly localised about the thermocline extending down to $1000 \mathrm{~m}$ depth and that $\mathrm{T}$ and $\mathrm{S}$ are highly correlated indicating that the fundamental T-S relationship is well preserved by this methodology.

Consider the local forecast error anomaly centred on $38^{\circ} 85^{\prime} \mathrm{S}, 153^{\circ}$ E. Figures $4(\mathrm{~g}-\mathrm{i})$ and $5(\mathrm{~g}-\mathrm{i})$ show the coincidence with the evolved BV averages in $\eta, T$ and $S$. This anomaly is noticeably absent in the ensemble forecast error. One may identify additional regions of substantial coincidence between the BV structures and forecast errors, which we interpret to be additional local regions of large dynamic instability. The day 28 ensemble forecast errors (four members) have significantly smaller amplitudes and contracted vertical structures as compared to the control forecast errors. For this case the RMS 

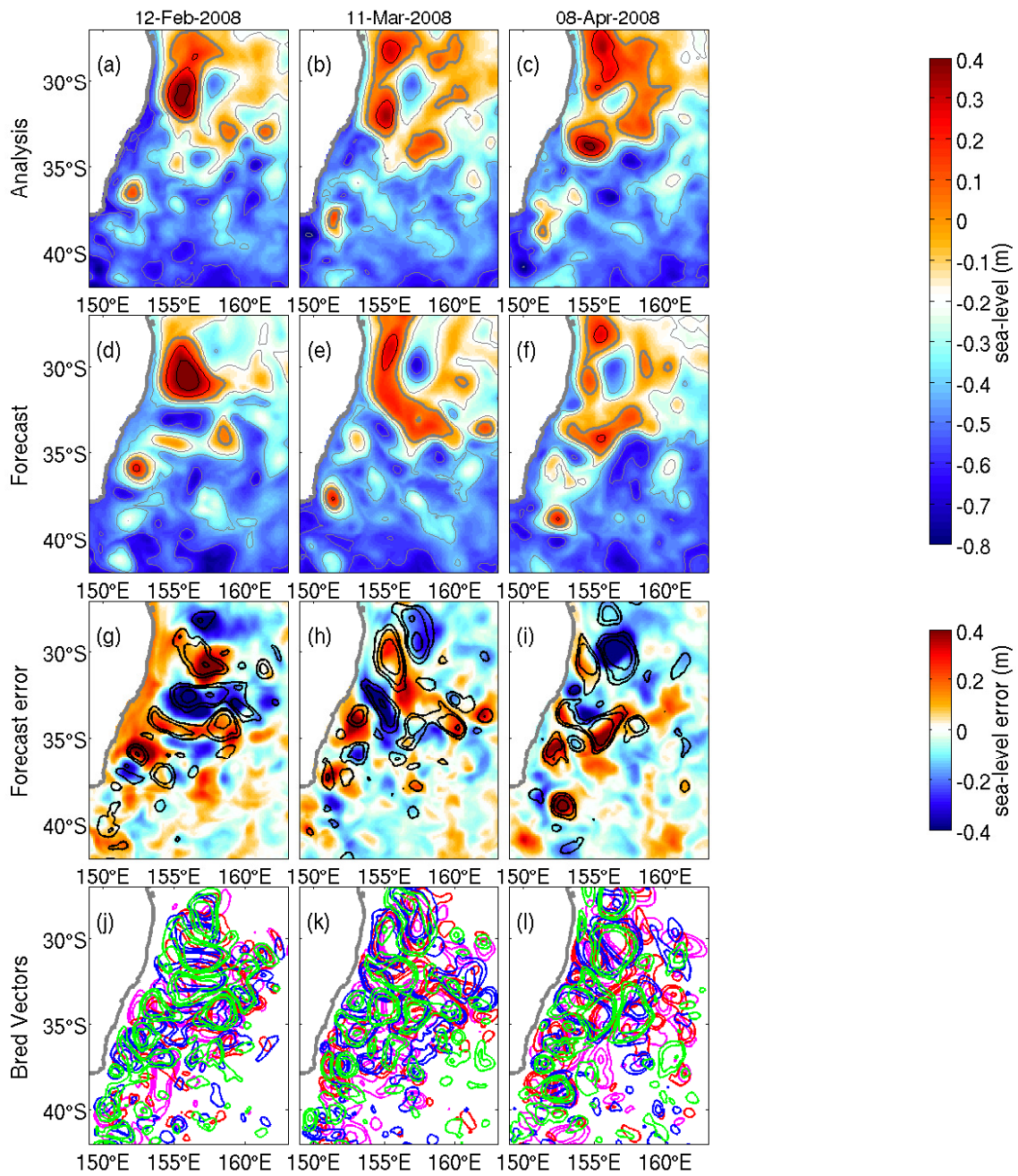

Figure 2: Surface height on T cells. Columns 1, 2, and 3 depict results valid for the 12th February, 11th March, and 8th April 2008 respectively. Row 1 figures a, b, and c; Analysis fields, Row 2 figures e, f, and g; day 28 control forecasts. Row 3 figures i, j, and k; Comparison of ensemble averaged (four members) bred vectors $( \pm 0.4, \pm 0.25$ and $\pm 0.15 \mathrm{~m}$ contours) and day 28 forecast error (shaded) valid for the i) 12th February, j) 11th March, and k) 8th April 2008 respectively. Row 4 figures $\mathrm{m}, \mathrm{n}$, and $\mathrm{o} ; \pm 0.4, \pm 0.25$ and $\pm 0.15 \mathrm{~m}$ contours for each of the four individual bred vectors (green, blue, red and magenta). 


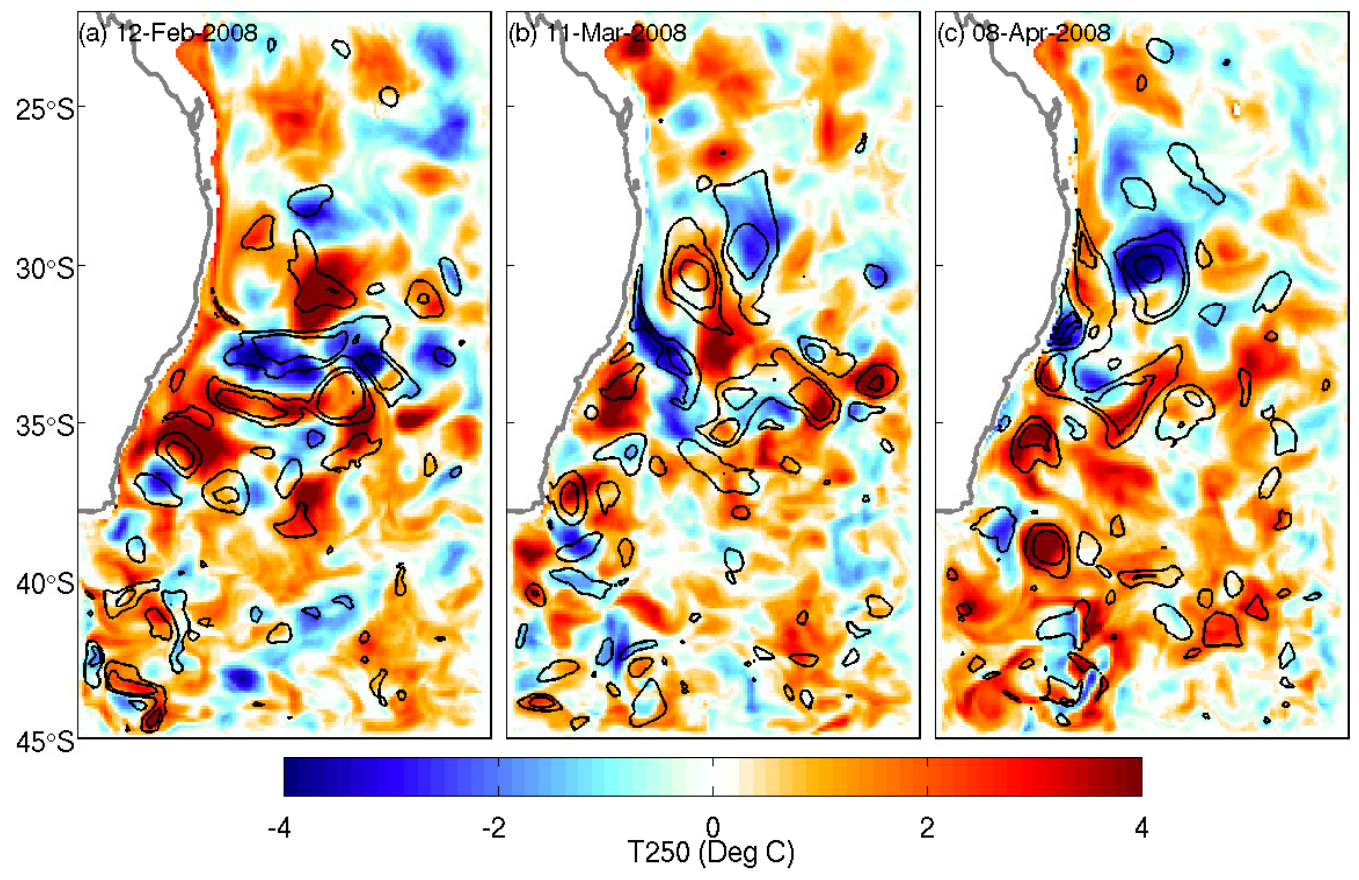

Figure 3: Comparison of ensemble averaged evolved bred vectors (four members contours) and forecast error (shaded) for T250 at monthly intervals over a period beginning on the 12 th February 2008. Contours shown are $\pm 3.5^{\circ} \mathrm{C}$, $\pm 2.0^{\circ} \mathrm{C}$ and $\pm 1.0^{\circ} \mathrm{C}$. 
scores for the four member ensemble forecast are noticeably better than the control, and the ensemble forecast error is largely absent throughout the length of the EAC extension (below $32^{\circ} 5^{\prime} \mathrm{S}$ ).

\section{Discussion and conclusions}

We conducted an ensemble prediction study of the EAC on timescales of one month, focussing on the quantification of forecast errors through validation with analysis fields. We employed bred perturbation vectors to identify the spatial patterns of the fastest growing errors for a given initial state, where the initial state is an analysis produced via an ensemble optimal interpolation data assimilation scheme. As a severe test of the method we considered the Austral summer and autumn, seasons corresponding to the largest eddy variability. We demonstrated that individual perturbations, while globally distinct, have ensemble averages that correspond to the observed regions of significant dynamic instability and where forecast errors are large. In particular, these regions are associated with the Tasman front and the EAC extension. Our results show that perturbation vectors generated from the past that are well embedded in the attractor, such as BVs, are an effective, computationally inexpensive means to calculate flow dependent information critical to accurate forecasting. Further, a very small BV ensemble may be run to identify regions where additional observations may be targeted.

Acknowledgements It is a pleasure to acknowledge computational assistance from Dr Justin Freeman, BoM. 


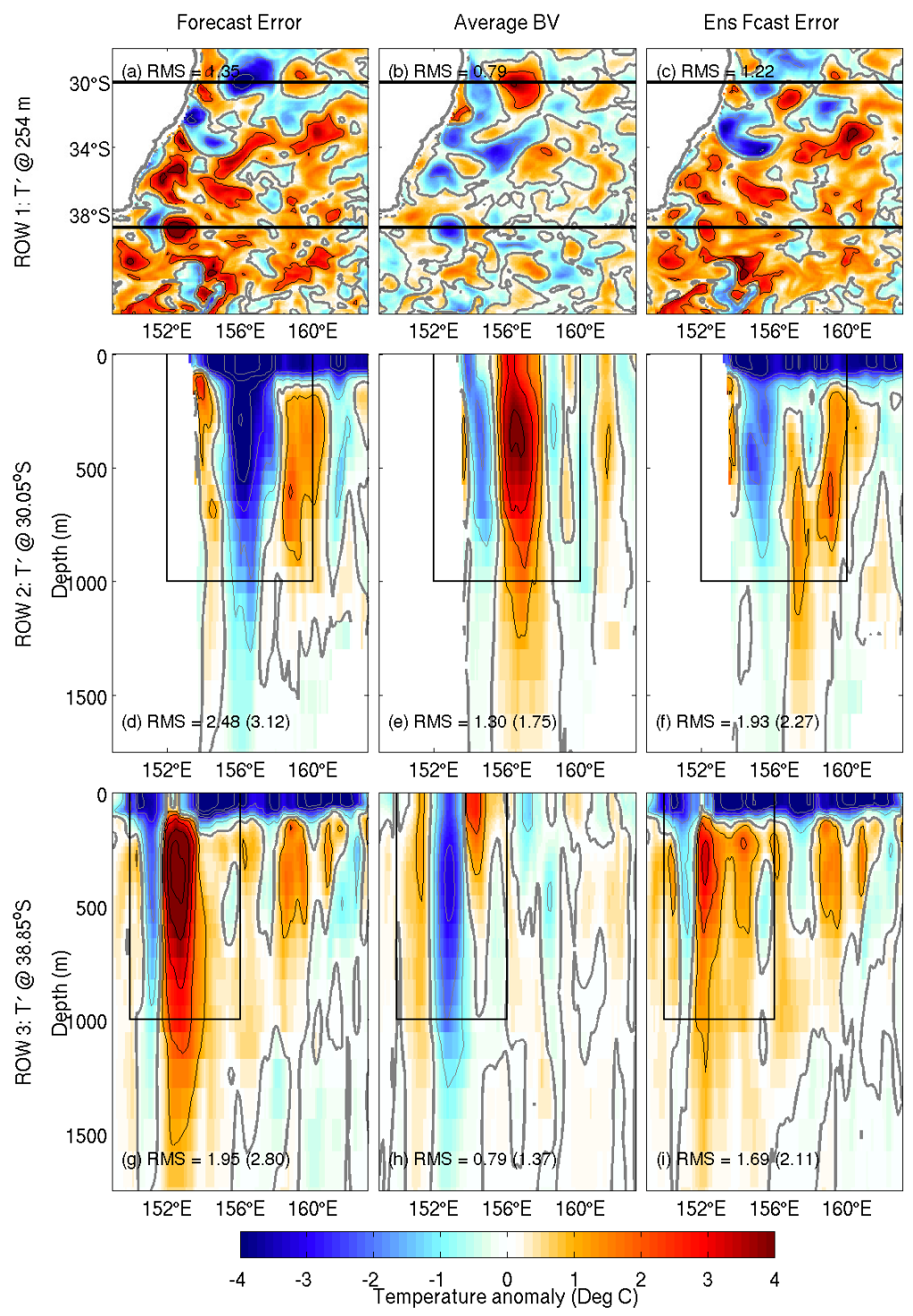

Figure 4: Comparison of day 28 forecast error (left), ensemble averaged bred vectors (middle) and ensemble averaged forecast error (right) (four members) valid on the 8th April; Showing T250 (a-c) and vertical sections along $35.55^{\circ} \mathrm{S}$ $(\mathrm{d}-\mathrm{f})$ and $38.85^{\circ} \mathrm{S}(\mathrm{g}-\mathrm{i})$. RMS errors are shown for the entire domain and for subdomains encompassing the dominant coherent error structures. 

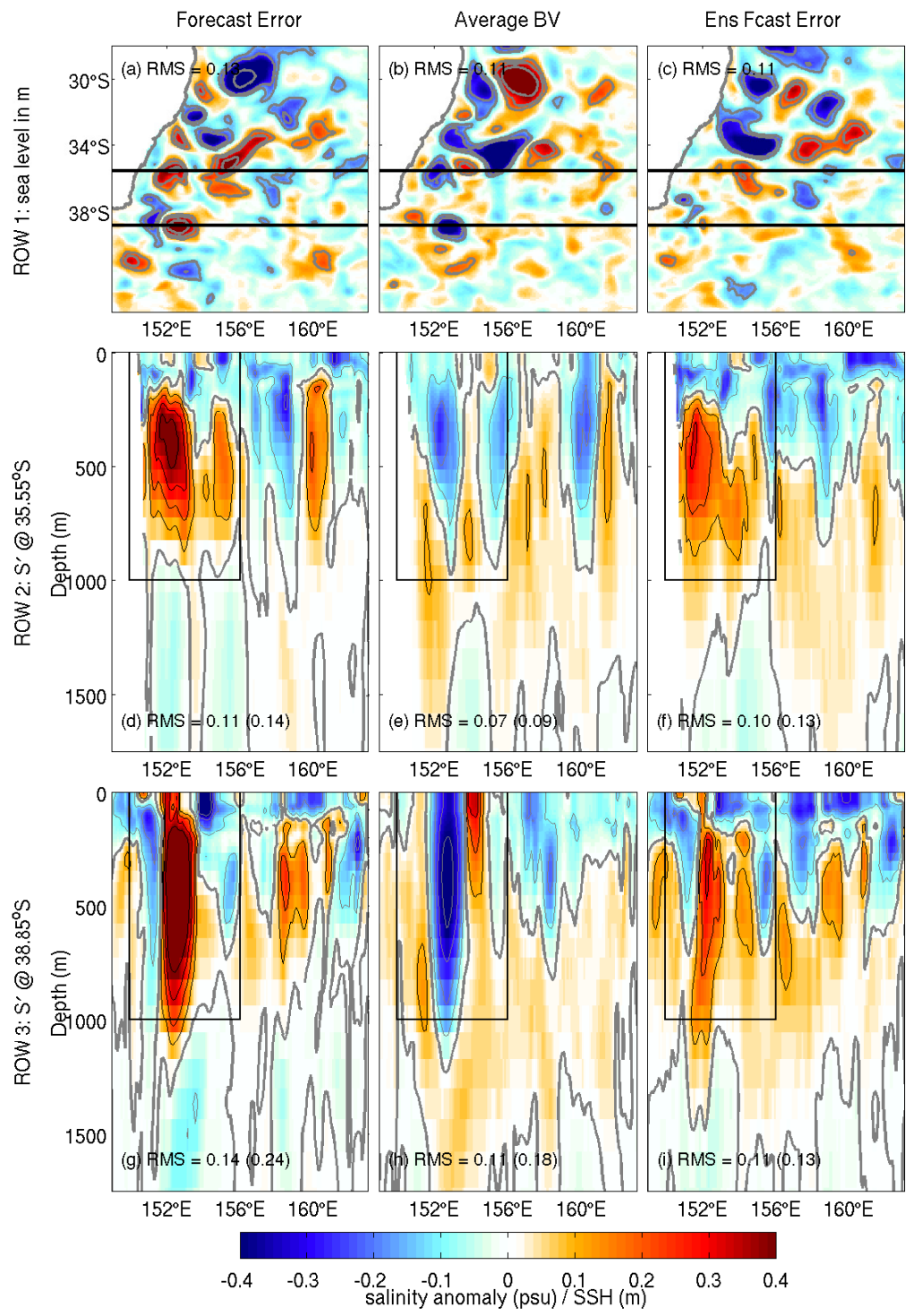

FiguRE 5: Comparison of day 28 forecast error (left), ensemble averaged bred vectors (middle) and ensemble averaged forecast error (right) (four members) valid on the 8th April; Showing SSH $(\mathrm{a}-\mathrm{c})$ and salinity vertical sections along $30.05^{\circ} \mathrm{S}(\mathrm{d}-\mathrm{f})$ and $38.85^{\circ} \mathrm{S}(\mathrm{g}-\mathrm{i})$. RMS errors are shown for the entire domain and for subdomains encompassing the dominant coherent error structures. 


\section{References}

[1] G. R. Brassington, P. R. Oke, and T. Pugh, BLUElink Operational ocean prediction in Australia, vol. 5, ch. 11, pp. 87-95, World Scientific Publishing, 2006. C43

[2] M. Corazza, E. Kalnay, D. J. Patil, S.-C Yang, R. Morss, M. Cai, I. Szunyogh, B. R. Hunt, and J. A. Yorke, Use of the breeding technique to estimate the structure of the analysis errors of the day, Nonlin. Proc. in Geophys. 10 (2003), 233-243.

http://hal . archives-ouvertes.fr/hal-00302201/ C46

[3] S. M. Griffies, M. J. Harrison, R. C. Pacanowski, and A. Rosati, A technical guide to MOM4 GFDL ocean technical group report no 5, 2003. http://citeseerx.ist.psu.edu/viewdoc/download?doi=10.1.1. 143. 8321\&rep=rep1\&type=pdf $\mathrm{C} 43$

[4] H. E. Hurlburt, G. B. Brassington, Y. Drillet, M. Kamachi, M. Benkiran, R. Bourdalle-Badie, E. P. Chassignet, G. A. Jacobbs, O. Le Galloudec, J.-M. Lellouche, E. J. Metzger, P. R. Oke, T. F. Pugh, A. Schiller, O. M. Smedstad, B. Tranchant, H. Tsujino, N. Usui and A. J. Walcraft, High-resolution global and basin-scale ocean analyses and forecasts., Oceanography 22 (2009), no. 3, 80-97. http://handle.dtic.mil/100.2/ADA508067 C39

[5] M. M. Mata, M. Tomczak, S. Wijffels, and J. A. Church, East Australian Current volume transports at 30S: Estimates from the World Ocean Circulation Experiment hydrographic sections PR11/P6 and the PCM3 current meter array., J. Geophys. Res. 105 (2000), 28, 509-28, 526. doi:10.1029/1999JC000121 C41

[6] M. M. Mata, S. E. Wijffels, J. A. Church, and M. Tomczak, Eddy shedding and energy conversion in the East Australian Current, J. Geophys. Res. 111 (2006), C09034. doi:10.1029/2006JC003592 C41 
[7] T. J. O'Kane and J. S. Frederiksen, Statistical dynamical subgrid-scale parameterizations for geophysical flows, Physica Scripta T132 (2008), 014033. doi:10.1088/0031-8949/2008/T132/014033 C44

[8] T. J. O'Kane, P. R. Oke and P. A. Sandery, Ocean Modelling, to appear (2011). doi:10.1016/j.ocemod.2011.04.003 C40

[9] P. R. Oke, M. Balmaseda, M. Benkiran, J. A. Cummings,

E. Dombrowsky, Y. Fujii, S. Guinehut, G. Larnicol, P.-Y. Le Traon, and M. J. Martin, Observing System Evaluations using GODAE systems, Oceanography (2009), 144-153.

http://handle.dtic.mil/100.2/ADA511325. C44

[10] P. R. Oke and D. A. Griffin, The cold-core eddy and strong upwelling off the coast of New South Wales in early 2007, Deep Sea Research 58 (2011), 574-591. doi:10.1016/j.dsr2.2010.06.006 C41

[11] D. Pazó, M. A. Rodríguez, and J. M. López, Spatio-temporal evolution of perturbations in ensembles initialized by bred, Lyapunov and singular vectors, Tellus 62A (2010), 10-23. doi:10.1111/j.1600-0870.2009.00419.x $\mathrm{C} 40$

[12] P. A. Sandery, G. B. Brassington, and J. Freeman, Adaptive nonlinear dynamical initialization., J. Geophys. Res. 116 (2011), C01021. doi:10.1029/2010JC006260 C43

[13] A. Schiller, P. R. Oke, G. B. Brassington, M. Entel, R. Fiedler, D. A. Griffin, and J.V. Mansbridge, Eddy-resolving ocean circulation in the Asian-Australian region inferred from an ocean reanalysis effort., Progress in Oceanography 76 (2008), 334-365. doi:10.1016/j.pocean.2008.01.003 C42

[14] Z. Toth and E. Kalnay, Ensemble forecasting at NMC, Bull. Amer. Meteor. Soc. 74 (1993), 2317-2330. doi:10.1175/1520-0477(1993)074;2317:EFANTG_2.0.CO;2 C40 


\section{Author addresses}

1. T. J. O'Kane, Centre for Australian Weather and Climate Research, CSIRO Marine and Atmospheric Research, Hobart, Tasmania, Australia.

mailto:terence.okane@csiro.au

2. P. R. Oke, Centre for Australian Weather and Climate Research, CSIRO Marine and Atmospheric Research, Hobart, Tasmania, Australia.

3. P. A. Sandery, Centre for Australian Weather and Climate Research, Bureau of Meteorology, Docklands, Victoria, Australia. 